\title{
Desempenho de fungicidas no controle da mancha-de-macrospora na cultura do milho
}

\author{
Daiana Bampi ${ }^{1}$, Ricardo Trezzi Casa ${ }^{2}$, Amauri Bogo², Luis Sangoi², Cristiano Sachs ${ }^{3}$, Jonatha Marcel Bolzan ${ }^{4}$ \& \\ Giovani Piletti ${ }^{3}$
}

\begin{abstract}
${ }^{1}$ Doutoranda do Curso de Proteção de Plantas, Universidade Estadual Paulista Júlio de Mesquita Filho, UNESP, Câmpus de Botucatu, SP. Av. Universitária, 3329. Bairro: Altos do Paraiso. Cep: 18610-031, Botucatu, SP.; ${ }^{2}$ Professores do Departamento de Agronomia, Universidade do Estado de Santa Catarina, UDESC, Lages, SC; ${ }^{3}$ Mestrandos em Produção Vegetal, UDESC, Lages, SC; ${ }^{4}$ Eng. Agr. Syngenta, Tangará da Serra, MT. Autor para correspondência: Daiana Bampi (daianabampi@yahoo.com.br)

Data de chegada: 20/08/2012. Aceito para publicação em: 19/11/2012.
\end{abstract}

\section{RESUMO}

Bampi, D.; Casa, R.T.; Bogo, A.; Sangoi, L.; Sachs, C.; Bolzan, J.M.; Piletti, G. Desempenho de fungicidas no controle da mancha-de-macrospora na cultura do milho. Summa Phytopathologica, v.38, n.4, p.319-322, 2012.

A aplicação de fungicidas é uma das estratégias de controle de doenças fúngicas foliares do milho. Não existem, no Brasil, fungicidas registrados para controle da mancha-de-macrospora (MFS). O objetivo deste trabalho foi avaliar a eficácia de 16 fungicidas no controle da MFS de forma protetora, curativa e erradicante. Utilizaramse fungicidas dos grupos químicos dos benzimidazóis, estrobilurinas e triazóis, isolados ou em misturas, em delineamento experimental inteiramente casualizado, com seis repetições de cinco plantas, totalizando 30 plantas por tratamento. Os experimentos foram conduzidos em casa-de-vegetação, com o híbrido simples AS 1565, com as plantas nos estádios fenológicos de duas a seis folhas expandidas, utilizando um isolado de Stenocarpella macrospora do mesmo híbrido. Para a ação preventiva, curativa e erradicante depositou-se o inóculo no cartucho das plantas 48 horas após, 48 horas antes e dez dias antes da aplicação dos fungicidas, respectivamente. O experimento foi repetido duas vezes. Os dados foram submetidos à análise de variância $(\mathrm{p} \leq 0,05)$, e as médias dos tratamentos comparadas pelo teste de Scott-Knott $(\mathrm{p} \leq 0,05)$. A severidade foliar estimada da doença foi determinada 21 dias após as inoculações. Todos os fungicidas difereriram significativamente do tratamento testemunha na ação preventiva, curativa e erradicante. $\mathrm{Na}$ ação preventiva o controle médio da doença foi de $85 \%$. As misturas de triazóis + estrobilurinas controlaram em média $75 \%$ a severidade da doença, enquanto que os produtos isolados como as estrobilurinas reduziram $62 \%$, os benzimidazóis $55 \%$ e os triazóis $38 \%$ na ação curativa. O menor controle foi obtido na ação erradicativa com redução média de $40,1 \%$ da severidade da doença, não havendo diferença significativa entre fungicidas.

Palavras-chave adicionais: Controle químico, diplodia, Stenocarpella macrospora, Zea mays

\section{ABSTRACT}

Bampi, D.; Casa, R.T.; Bogo, A.; Sangoi, L.; Sachs, C.; Bolzan, J.M.; Piletti, G. Fungicide performance on the control of macrospora leaf spot in corn. Summa Phytopathologica, v.38, n.4, p.319-322, 2012.

Fungicide application is one of the control strategies of fungal diseases in corn leaves. In Brazil, there are no fungicides recorded for the control of corn macrospora leaf spot (MLS). The aim of this study was to assess the efficacy of 16 fungicides on MLS control in a protective, curative and eradicant form. Fungicides of the chemical groups of benzimidazoles, strobilurins and triazoles were used alone or in mixture, in completely randomized block design, with six replicates of five plants, totaling 30 plants per treatment. The experiments were carried out in a greenhouse with the single-cross hybrid AS 1565 in phenological stage of two to six expanded leaves, using an isolate of $S$. macrospsora from the same hybrid. The inoculum was deposited into the cartridge of plants at 48 hours after, 48 hours before and 10 days before fungicide applications for preventive, curative and eradicant action, respectively. The experiment was repeated twice. The data underwent analysis of variance $(p \leq 0.05)$, and the means of treatments were compared by using the Scott-Knott test $(\mathrm{p} \leq 0.05)$. Severity was estimated at 21 days after inoculations. All fungicides significantly differed from the control treatment in the preventive, curative and eradicant action. For the preventive action, mean disease control was $85 \%$. The mixture of triazoles plus strobilurins controlled, on average, $75 \%$ of the disease severity, while the isolated products such as strobilurins reduced it by $62 \%$, benzimidazoles by $55 \%$ and triazoles by $38 \%$ for the curative action. The lowest control was obtained for the eradicant action, with mean reduction of $40.1 \%$ in the disease severity and no significant difference among fungicides. 
A mancha-de-macrospora (MFS), causada pelo fungo necrotrófico Stenocarpella macrospora (Earle) Sutton [Sin. Diplodia macrospora Earle], tem ocorrido com frequência na cultura do milho (Zea mays L.) nos últimos anos no Brasil principalmente devido ao monocultivo em sistema plantio direto e ao aumento da área de milho safrinha $(5,22)$. Além de mancha foliar, o patógeno pode provocar podridão de colmo, podridão de espiga e grãos ardidos $(15,17,21)$. Nas lesões foliares pode haver produção de inóculo suficiente para infectar a base da espiga, reduzindo a produtividade e a sanidade de grãos $(2,9)$.

Informações sobre resistência genética de híbridos de milho específica para MFS são escassas $(4,5,11)$. As principais estratégias de controle tem se concentrado no manejo por práticas culturais, principalmente a rotação de culturas (6).

Ênfase tem sido dado ao controle de doenças foliares do milho pela aplicação de fungicidas, o que tem proporcionado a redução dos sintomas e incremento na produtividade em relação a áreas não tratadas $(7,12,13,22)$.

Entre os fungicidas disponíveis no mercado brasileiro para o controle de doenças foliares do milho, os penetrantes móveis pertencentes aos grupos químicos dos triazóis (inibidores da síntese de esteróis - ISE) e das estrobilurinas (inibidores da respiração mitocondrial - IQe), são os mais utilizados isoladamente ou em misturas pré-fabricadas. Os benzimidazóis (inibidores da divisão celular) também são utilizados, porém com menor escala. Esses fungicidas podem ter ação protetora, curativa e erradicativa na planta, sendo a ação preventiva realizada no estágio de pré-infecção. Nesse caso o fungicida inibe a germinação ou impede a penetração do fungo nos tecidos do hospedeiro. A ação curativa tem efeito pós-infecção e pré-sintoma. A ação erradicativa envolve o efeito dos fungicidas no estágio pós-sintoma (16).

De acordo com o Ministério da Agricultura são inexistentes informações de fungicidas indicados para o controle específico da MFS (10). Para tanto, trabalhos com controle químico visando o controle dessa doença devem ser explorados, principalmente quantificando a eficácia dos fungicidas em relação aos modos de ação e a reação de híbridos em diferentes condições de ambiente. Este trabalho foi conduzido com o objetivo de avaliar a performance de fungicidas no controle protetor, curativo e erradicativo de $S$. macrospora em plantas de híbrido de milho suscetível.

\section{MATERIAL E MÉTODOS}

O experimento foi conduzido em casa-de-vegetação na Universidade do Estado de Santa Catarina - UDESC, no município de Lages, SC, no ano de 2011 , com temperatura de $25^{\circ} \mathrm{C} \pm 3{ }^{\circ} \mathrm{C}$ e umidade relativa do ar aproximada de $70 \%$, utilizando o híbrido simples de milho AS 1565 e um isolado do fungo do mesmo híbrido oriundo de Lages, SC.

Para o isolamento e preparo da suspensão de inóculo, pedaços de $10 \mathrm{~cm}$ de colmos naturalmente infectados por S. macrospora foram lavados, desinfestados com hipoclorito $(1,0 \%)$ e submetidos à câmara úmida com temperatura de $30{ }^{\circ} \mathrm{C}$ por um período de 48 horas até a extrusão de cirro de conídios. Raspagem com pincel para remoção dos cirros foram realizadas com água destilada e esterilizada + duas gotas por litro do surfactante Tween 20 (polioxietilenosorbitano).

Os fungicidas testados (g ou $\mathrm{mL}$ i.a.ha ${ }^{-1}$ ) foram: azoxistrobina $(62,5)$ (Priori $250 \mathrm{SC}$ ), trifloxistrobina (125) (Flint $500 \mathrm{WG}$ ), piraclostrobina (150) (Comet $250 \mathrm{CE}$ ), tebuconazol (200) (Folicur 200 EC), propiconazol (100) (Tilt $250 \mathrm{CE}$ ), epoxiconazol $(93,75)$ (Opus 125 SC), tetraconazol (50) (Domark $100 \mathrm{CE}$ ), metconazol
$(81,08)$ (Caramba $90 \mathrm{CE})$, ciproconazol (30) (Alto $100 \mathrm{CE}$ ), carbendazim (250) (Derosal $500 \mathrm{SC})$, tiofanato metílico $(267,9)$ (Tiofanato metílico $800 \mathrm{WG})$, tiabendazol $(247,8)$ (Tecto $484 \mathrm{SC})$, azoxistrobina + ciproconazol $(60+24)$ (Priori Xtra 280 SC), piraclostrobina + epoxiconazol $(100+37,5)($ Opera $283 \mathrm{SE})$, trifloxistrobina + tebuconazol $(75+150)$ (Nativo $300 \mathrm{SC}$ ), picoxistrobina + ciproconazol $(60+24)$ (Aproach Prima 280 SC). A escolha das doses foi decorrente do registro do fungicida para cultura.

Foram semeadas oito sementes em vaso de 1,5 L contendo uma mistura homogênea de substrato $(80 \%)$ e vermiculita $(20 \%)$. Os vasos foram mantidos em casa-de-vegetação e irrigados de acordo com as necessidades hídricas da cultura. No estádio fenológico V2 (duas folhas totalmente expandidas) foi realizado o desbaste, deixando-se cinco plantas por vaso. Os fungicidas foram aplicados com pulverizador costal com pressão gerada por gás $\mathrm{CO}_{2}$ numa vazão de $200 \mathrm{~L} \mathrm{ha}^{-1}$. Tratamento testemunha aplicou-se somente água. As inoculações foram feitas depositando-se no cartucho de cada planta nos estádios V2 a V3 (duas a três folhas totalmente expandidas), $0,2 \mathrm{~mL}$ de uma suspensão de $7 \times 10^{4}$ conídios $\mathrm{mL}^{-1}$ com pipetador automático.

$\mathrm{Na}$ ação preventiva, a inoculação foi feita 48 horas após a pulverização dos fungicidas. No controle curativo, a inoculação foi realizada 48 horas antes da pulverização. No controle erradicativo, a pulverização foi feita nos estádios V4 a V5, 10 dias após a inoculação, com sintomas presentes. As plantas foram mantidas por $48 \mathrm{~h}$ em ambiente com umidade relativa do ar próxima de $100 \%$ por meio de pulverização de água a cada 6 horas. Vinte e um dias após as inoculações, quando o milho estava entre os estádios fenológicos V5 e V6 (cinco a seis folhas totalmente expandidas), a quarta folha expandida de cada planta foi coletada. Mediu-se a área clorótica e necrótica das lesões com um paquímetro digital para obtenção da severidade da doença em função da área foliar (A) que foi estimada aplicando-se a expressão A $=\mathrm{C}$ (comprimento) $\mathrm{L}$ L (largura) x 0,75 (coeficiente de correção) (19).

$\mathrm{O}$ delineamento experimental foi inteiramente casualizado, com seis repetições, sendo cada repetição constituída por um vaso com cinco plantas, totalizando 30 plantas por tratamento. $\mathrm{O}$ experimento foi repetido duas vezes.

Os dados foram submetidos à análise da variância $(p \leq 0,05)$. A comparação entre as médias dos tratamentos foi procedida por meio do teste de Scott-Knott $(\mathrm{p} \leq 0,05)$, utilizando o software R (14).

\section{RESULTADOS E DISCUSSÃO}

Os fungicidas testados de forma preventiva reduziram significativamente a área foliar afetada pela MFS quando comparados à testemunha (Tabela 1). Não houve diferença entre tratamentos com exceção daquele com tetraconazol que apresentou severidade mais elevada em relação os tratamentos com demais fungicidas (Tabela 1). Os fungicidas do grupo químico dos benzimidazóis apresentaram melhor controle preventivo, com $91,1 \%, 90,1 \%$ e $88,2 \%$, respectivamente para carbendazim, tiofanato metílico e tiabendazol. A aplicação de fungicidas de forma preventiva controlou $85 \%$ em média a severidade da MFS em relação à testemunha.

Quanto a ação curativa todos os fungicidas diferiram da testemunha reduzindo a severidade (Tabela 1). Os fungicidas mais eficientes foram as misturas de estrobilurinas + triazóis [piraclostrobina + epoxiconazol $(80,1 \%)$, azoxistrobina + ciproconazol $(78,5 \%)$, picoxistrobina + ciproconazol $(72,1 \%)$ e trifloxistrobina + tebuconazol $(70,6 \%)]$ diferindo dos demais fungicidas testados. A mistura de estrobilurinas + triazóis controlou em média $75 \%$ a severidade da MFS, enquanto 
Tabela 1. Ação preventiva, curativa e erradicante de fungicidas sobre o fungo Stenocarpella macrospora em folhas de plantas de milho do híbrido simples AS 1565. Lages, SC, 2011

\begin{tabular}{|c|c|c|c|c|c|c|c|}
\hline \multirow[b]{2}{*}{ Tratamento } & \multirow[b]{2}{*}{ g i.a. ha h $^{-1}$} & \multicolumn{2}{|c|}{ Preventiva } & \multicolumn{2}{|c|}{ Curativa } & \multicolumn{2}{|c|}{ Erradicante } \\
\hline & & $\begin{array}{c}\text { Severidade } \\
(\%)\end{array}$ & $\begin{array}{c}\text { Controle } \\
(\%)\end{array}$ & $\begin{array}{c}\text { Severidade } \\
(\%)\end{array}$ & $\begin{array}{c}\text { Controle } \\
(\%)\end{array}$ & $\begin{array}{c}\text { Severidade } \\
(\%)\end{array}$ & $\begin{array}{c}\text { Controle } \\
(\%)\end{array}$ \\
\hline Carbendazim & 250 & $0,99 \mathrm{c}$ & 91,1 & $8,33 \mathrm{c}$ & 62,9 & $15,04 \mathrm{~b}$ & 34,4 \\
\hline Tiabendazol & 247,8 & $1,31 \mathrm{c}$ & 88,2 & $12,10 \mathrm{~b}$ & 46,1 & $13,07 \mathrm{~b}$ & 43,0 \\
\hline Tiofanato metílico & 267,9 & $1,10 \mathrm{c}$ & 90,1 & $9,78 \quad \mathrm{c}$ & 56,4 & $16,06 \mathrm{~b}$ & 29,9 \\
\hline Epoxiconazol & 93,7 & $1,72 \mathrm{c}$ & 84,6 & $15,40 \mathrm{~b}$ & 31,4 & $14,66 \mathrm{~b}$ & 36,1 \\
\hline Metconazol & 81,1 & $2,00 \mathrm{c}$ & 82,1 & $15,16 \mathrm{~b}$ & 32,5 & $13,74 \mathrm{~b}$ & 40,1 \\
\hline Propiconazol & 100 & $1,41 \mathrm{c}$ & 87,3 & $8,36 \mathrm{c}$ & 62,7 & $14,69 \mathrm{~b}$ & 35,9 \\
\hline Tebuconazol & 200 & $2,06 \mathrm{c}$ & 81,5 & $12,73 \mathrm{~b}$ & 43,3 & $12,64 \mathrm{~b}$ & 44,9 \\
\hline Tetraconazol & 50 & $3,48 \mathrm{~b}$ & 68,8 & $15,40 \mathrm{~b}$ & 31,4 & $12,66 \mathrm{~b}$ & 44,8 \\
\hline Azoxistrobina + ciproconazol & $60+24$ & $1,34 \mathrm{c}$ & 88,0 & $4,81 \mathrm{~d}$ & 78,5 & $13,27 \mathrm{~b}$ & 42,1 \\
\hline Picoxistrobina + ciproconazol & $60+24$ & $1,57 \mathrm{c}$ & 85,9 & $6,31 \mathrm{~d}$ & 72,1 & $15,01 \mathrm{~b}$ & 34,5 \\
\hline Piraclostrobina + epoxiconazol & $100+37,5$ & $1,76 \mathrm{c}$ & 84,2 & $4,50 \mathrm{~d}$ & 80,1 & $13,99 \mathrm{~b}$ & 39,0 \\
\hline Trifloxistrobina + tebuconazol & $75+150$ & $1,98 \mathrm{c}$ & 82,2 & $6,60 \mathrm{~d}$ & 70,6 & $12,71 \mathrm{~b}$ & 44,5 \\
\hline Média & & & 85,0 & & 55,03 & & 40,1 \\
\hline C.V. $(\%)$ & & 28,26 & & 27,91 & & 14,77 & \\
\hline
\end{tabular}

*Médias seguidas da mesma letra na coluna não diferem entre si pelo Teste de Scoot knott a 5\%.

que os ingredientes ativos do grupo das estrobilurinas reduziram $62 \%$, dos benzimidazóis $55 \%$ e dos triazóis $38 \%$.

Todos os fungicidas diferiram da testemunha quando aplicados de forma erradicativa reduzindo em média $40,1 \%$ a severidade da doença, porém não houve diferença significativa entre tratamentos (Tabela 1).

Em aplicação preventiva no híbrido 2B710, Duarte et al. (7) reportam redução média de $72 \%$ na severidade da MFS aos 56 dias após aplicação dos fungicidas ( $\mathrm{g}$ ou $\mathrm{mL}$.i.a ha $\mathrm{ha}^{-1}$ ) azoxistrobina + ciproconazol $(60+24)$ (Priori Xtra 280 SC), tebuconazol (200) (Folicur 200 EC), carbendazim (250) (Derosal 500 SC), tetraconazol (50) (Domark $100 \mathrm{CE}$ ), azoxistrobina (75) (Priori $250 \mathrm{SC}$ ) e piraclostrobina + epoxiconazol $(100+37,5)($ Opera $283 \mathrm{SE})$.

A aplicação de fungicidas de forma preventiva, quando comparada à curativa e à erradicativa, foi mais eficaz no controle da doença, independentemente do produto utilizado. Neste caso, a redução da severidade pode ser atribuída à ação fungitóxica dos fungicidas sobre a germinação dos esporos do fungo, inibindo a germinação do mesmo e por conseguinte, o processo de infecção. De acordo com Juliatti et al. (8), a prevenção é a melhor forma de controle, além de contribuir para reduzir risco de aparecimento de isolados resistentes aos fungicidas. Por outro lado, a utilização de misturas de fungicidas com modo de ação diferente apresentou melhor eficácia no controle curativo da MFS, uma vez que esta pode atuar em diferentes sítios de desenvolvimento do patógeno, reduzindo assim a probabilidade de ocorrência de populações resistentes do fungo. Além disso, segundo Reis et al. (16), a mistura de fungicidas poderá aumentar o período de proteção e o espectro de ação sobre outros fungos patogênicos que causam doenças foliares do milho, como ferrugens, cercosporiose e helmintosporioses, e também contribuir para evitar os problemas com a seleção de linhagens resistentes de fungos.
$\mathrm{Na}$ aplicação de forma curativa e erradicativa houve desenvolvimento de infecção antes da aplicação dos fungicidas. Esse fato contribuiu para maior severidade da doença principalmente na ação erradicativa, pois neste caso, estava mais avançada a colonização do fungo nos tecidos da planta, o que proporcionou menor controle da doença em relação à aplicação preventiva. Bampi et al. (1) e Casa et al. (3), observaram, no campo, com o mesmo híbrido AS 1565, dificuldade de controle erradicativo da MFS com uma pulverização em folhas sintomáticas com as mesmas quatro misturas de estrobilurinas + triazóis utilizadas neste trabalho. Esses autores descreveram uma redução média de $62,3 \%$ no tamanho das lesões e 58,25\% na formação de picnídios do fungo no tecido necrosado. Isto demonstra que a proteção da planta pela aplicação do fungicida antes da infecção de $S$. macrospora e/ou quando realizada de forma curativa, tende a retardar o início de uma epidemia no campo e diminuir a chance de ocorrência de populações do patógeno resistentes. A estratégia de aplicar fungicidas antes da penetração e colonização de patógenos, em áreas com alta pressão de inóculo e condições ambientais favoráveis ao desenvolvimento de epidemias, pode reduzir o progresso de doenças (18). A aplicação foliar de um fungicida penetrante móvel pode interromper o progresso da doença logo após a pulverização e perdurar por um período efetivo durante o qual o aumento da doença é mínimo (20).

Mesmo não havendo fungicidas registratos especificamente para à MFS, pode-se concluir neste trabalho que os fungicidas benzimidazóis (carbendazim, tiofanato metílico, tiabendazol) aplicados de forma preventiva e a mistura de estrobilurinas + triazóis (azoxistrobina + ciproconazol, trifloxistrobina + tebuconazol, piraclostrobina + epoxiconazol, picoxistrobina + ciproconazol) aplicados de forma curativa e erradicativa, apresentaram desempenho 
satisfatório no controle da doença. As misturas comerciais de estrobilurinas + triazóis devem ser preferencialmente indicadas, uma vez que, no campo, normalmente numa mesma planta e/ou folha têmse mais de uma doença, havendo necessidade de maior espectro de ação dos fungicidas. Além disto, os resultados quantitativos obtidos neste trabalho da eficácia dos fungicidas para o controle da MFS são relevantes para a assistência técnica quando da tomada de decisão quanto a aplicação de fungicidas dentro de um programa de manejo integrado de doenças foliares da cultura do milho.

\section{REFERÊNCIAS BIBLIOGRÁFICAS}

1. Bampi, D.; Casa R.T.; Blum, M.M.C.; Wordell Filho, J.A.; Valenini, G. Ação de fungicidas na expansão da mancha-de-macrospora do milho. In: Reunião Técnica Catarinense de Milho e Feijão, 8, 2011, Chapecó. Resumos... Chapecó: Epagri, 2011.

2. Bampi, D.; Casa R.T.; Wordell Filho, J.A.; Kuhnem J.R, P.R.; Piletti, G. Relação entre a mancha-de-macrospora na folha da espiga e o rendimento e a sanidade de grãos de milho. In: Reunião Técnica Catarinense de Milho e Feijão, 8, 2011, Chapecó. Resumos... Chapecó: Epagri, 2011.

3. Casa, R.T.; Bampi, D.; Gheler, A.; Sachs, C.; Andriolli, C.; Bogo, A. Efeito de fungicida na inibição da formação do picnídio de Stenocapella macrospora em lesões foliares. Tropical Plant Pathology, Lavras, v. 36, p. 625, 2011.

4. Casa, R.T.; Bampi, D.; Kuhnem Junior, P.R.; Sangoi, L.; Blum, M.M.; Wordell Filho.J.A. Mancha-de-macrospora do milho no Sul do Brasil. Revista Plantio Direto, Passo Fundo, v.126, p.1318,2011

5. Casa, R.T.; Reis, E. M.; Kuhnem Junior, P.R.; Hoffmann, L.L. Doenças do milho: guia de campo para identificação e controle. Lages: Graphel, 2010. 79p.

6. Casa, R.T.; Reis, E.M.; Zambolim, L. Doenças do milho causadas pelo gênero Stenocarpella. Fitopatologia Brasileira, Brasilia, DF, v.31, n.5, p. 427-439, 2006

7. Duarte, R.P.; Juliatti, F.C.; Freitas, P.T. Eficácia de diferentes fungicidas na cultura do milho. Biosciencia Journal, Uberlândia, v.25, n. 4, p. 101-111, 2009.

8. Juliatti, F.C.; Brandão, A.M.; Santos, J.A.; Luz, V.C da. Fungicidas na parte aérea da cultura do milho: evolução de doenças fúngicas, perdas, resposta de híbridos e melhora da qualidade da produção. Revisão Anual de Patologia de Plantas, Passo Fundo, v.15,
2007. 450p.

9. Koehler, B. Natural mode of entrance of fungi into maize ears and some symptoms that indicate infection. Journal Agricultural Research, Washington, DC, v.64, n.8, p. 421-442, 1942.

10. Brasil - Ministério da Agricultura Pecuária e Abastecimento (MAPA). Disponível em: http://extranet.agricultura.gov.br/ agrofit cons/principal agrofit cons. Acesso em : 06 de out. 2011.

11. Olatinwo, R.; Cardwell, K.; Menkir, A.; Deadman, M.; Julian, A. Inheritance of resistance to Stenocarpella macrospora (Earle) ear rot of maize in the mid-altitude Zone of Nigeria. European Journal of Plant Pathology, Dordrecht, v.105, n.6 , p.535543, 1999.

12. Pinto, N.F.J.A. Controle químico de doenças foliares em milho. Revista Brasileira de Milho e Sorgo, Sete Lagoas, v.3, n.1, p.134-138, 2004.

13. Pinto, N.F.J.A.; Angelis de, B.; Habe, M.H. Avaliação da eficiência de fungicidas no controle da cercosporiose (Cercospora zeaemaydis) na cultura do milho. Revista Brasileira de Milho e Sorgo, Sete Lagoas, v.3, n.1, p.139-145, 2004.

14. R Development Core Team (2009). R: A language and environment for statistical computing. R Foundation for Statistical Computing, Vienna, ISBN 3-900051-07-0, URL http://www.Rproject.org.

15. Reis, E.M.; Casa, R.T.; Bresolin, A.C.R. Manual de diagnose e controle de doenças do milho. 2. ed. Lages, Graphel. 2004. 144p

16. Reis, E.M.; Reis, A.C.; Carmona, M.A. Manual de fungicidas: guia para controle químico de doenças de plantas. 6. ed. Passo Fundo. Ed. Universidade de Passo Fundo, 2010. 226p.

17. Shurtleff, M.C. Compendium of corn diseases. St. Paul: APS, 1992. $105 \mathrm{p}$

18. Silva, J. C.; Meyer, M.C.; Coutinho, W.M.; Suassuna, N.D. Fungitoxicidade de grupos químicos sobre Myrothecium roridum in vitro e sobre a mancha-de-mirotécio em algodoeiro. Pesquisa Agropecuária Brasileira, Brasilia, DF, v.41, n.5, p.755-761, 2006.

19. Tollenaar, M. Is low plant density a stress in maize? Maydica, Bergamo, v. 37, p.305-311, 1992

20. Ward, J.M.J.; Laing, M.D.; Rijkenberg, F.H.J.; Frequency and timing of fungicides applications for the control of gray leaf spot in maize. Plant Disease, Sant Paul, v.81, n.1 p. 41-48, 1997.

21. White, D.G. Compendium of corn diseases. $3^{\text {rd }}$ Ed. St. Paul: APS, 1999. 78p.

22. Wordell Filho, J.A.; CASA, R.T. Doenças na cultura do milho. In: WORDELl FILHO, J.A.; ELIAS, H.T. A cultura do milho em Santa Catarina. Florianópolis: Epagri, 2010, p.207-273. 